\title{
Computer simulation of the microstructure and rheology of semi-solid alloys under shear
}

\author{
Michel Perez ${ }^{\dagger *}$, Jean-Charles Barbé ${ }^{\ddagger}, Z_{\text {Zoltan Neda }}^{\S}$, \\ Yves Bréchet ${ }^{\top}$ and Luc Salvo ${ }^{\dagger}$
}

October 30, 2018

† Laboratoire Génie Physique et Mécanique des Matériaux, UMR CNRS 5010, BP 46, 38402 Saint Martin d'Hères Cedex, France

$¥$ Laboratoire de Solidification et de ses Procédés, DTA/SPCM, CEA Grenoble, 17 rue des Martyrs, 38054 Grenoble Cedex 9, France

$\S$ Babes-Bolyai University, Faculty of Physics, Dept. of Theoretical Physics, RO-3400 Cluj-Napoca, Romania

ฯ Laboratoire de Thermodynamique et de Physico-Chimie Métallurgique, UMR CNRS 5614, BP 75, 38402 Saint Martin d'Hères Cedex, France

Keywords: Casting, Semi-solid alloys, Theory and modeling

\begin{abstract}
The rheological behavior of metallic alloys containing both solid and liquid phases is investigated in the low solid fraction range $(<50 \%)$. This behavior depends on both the solid fraction and the shear rate. The concept of Effective Volume Fraction (EVF) is used to decorrelate the influence of these two parameters. At high shear rate the slurry behaves like a suspension of hard spheres, whereas at lower shear rate, particles tend to aggregate in clusters, entrapping liquid and thus, increasing the EVF and the viscosity. A lattice model is introduced to simulate the aggregation / break-up processes within a slurry under shear. When the steady state is reached, the entrapped liquid fraction is calculated, leading to a viscosity estimation. Simulation results for the viscosity and 3D cluster structure are in good agreement with experimental results.
\end{abstract}

\section{Introduction}

Semi-solid slurries are characterized by the coexistence of solid and liquid phases. They are usually observed in alloys with two or more constituents for temperatures between the solidus and liquidus lines. The rheological properties of this dual phase state is of interest both for casting and for metal forming operations known as thixoforming [1].

*email: Michel.Perez@gpm2.inpg.fr 
When submitted to shear, the steady state viscosity decreases as the applied shear rate increases, reaching values of order $100 \mathrm{mPa} . \mathrm{s}$ [2].

This behavior is called rheofluidization and it is usually explained by the interaction between the solid particles. It is a very general phenomenon observed in suspensions, metalic alloys in the semi-solid state, colloids, latexes, etc. Their characteristic behavior is qualitatively interpreted as a competition between the aggregating (Coulomb attraction, Wan Der Waals forces, surface forces...) and break-up forces (shear). At low shear rate (typically lower than $1 \mathrm{~s}^{-1}$ ), individual particles can aggregate in "clusters", being able to form a more or less rigid network and the slurry is considered as a solid. At very high shear rate, the motion of the particles prevents the particle/particle bonding and leads to a more dispersed suspension behaving more like a fluid.

The concept of effective volume fraction introduced by Quemada [3] allows to relate the cluster characteristics to the viscosity of the mixture. The aim of the present paper is to build on this idea by considering the dynamics of cluster formation, and to derive a phenomenology for non-newtonian behaviour of semi-solid slurry, which is based on an understanding of the elementary phenomena governing the cluster dynamics.

The rheofluidization has been extensively studied (see for instance the work of Quemada [4). Figure 1 shows a typical shear rate dependence of the viscosity for an interacting particles suspension. Shear thickening that may occur for higher values of the shear rate [5] is not considered. The behavior at high shear rate when particles are well separated has been accurately described by Krieger [6]:

$$
\eta=\eta_{0}\left(1-\frac{\Phi}{\Phi_{M}}\right)^{-2.5 \Phi_{M}}
$$

$\Phi$ being the solid fraction of the suspension and $\Phi_{M}$ its maximum value (close packing). Below a given value $\dot{\gamma}_{s}$, an increase of the viscosity is observed. This can be interpreted as an increase in the effective volume fraction $\Phi_{\text {eff }}$, which takes into account both the solid and the entrapped liquid. $\Phi_{\text {eff }}$ replaces $\Phi$ in equation [1.

Jeffrey and Acrivos [7] underlined the notion of structure resulting from aggregation between solid particles: for the same solid fraction $\Phi$, different structure can lead to different viscosities. The structure depends itself on the shear rate. This statement is easily understandable for the two extreme limits of the shear rate: "percolating network" for low shear rate and "disperse suspension" for high shear rate. Between these two domains, the structure of diphasic solutions is not fully understood due to several reasons:

- Mean-field modeling fails to describe the dynamics of such slurries because it does not take into account the interaction between solid particles.

- 2D observations are useful but not sufficient because they do not show the real state of aggregation. In particular, 3D connectivity of the solid particles cannot be easily revealed. Serial cutting [8] allows 3D reconstruction, but is only tractable for relatively small zones.

- Difficulty of 3D observations: Small Angle Scattering is efficiently used [9] with colloids, but gives only a characteristic length-scale of the suspension. Moreover, it is limited to small solid particles (less than a few microns). A new observational technique is under development: synchrotron-radiation microtomography allowing $3 \mathrm{D}$ investigation by means of phase contrast of a relatively large part of a sample with a resolution of a few micrometers 10 . 
In a previous work [11, an analytical estimation of the characteristic radius of clusters was derived considering that aggregation and coalescence lead to spherical compact clusters. This radius was found to decrease with the shear rate as $\dot{\gamma}^{-4 / 7}$. In this paper we discuss the aggregation and break-up phenomena leading to more or less opened structures neglecting, this time, the densification of the resulting clusters.

Different approaches has been used to simulate the rheological behaviour of colloidal suspensions. Dynamic simulation tools such as nonequilibrium Brownian dynamics [12 and Stokesian dynamics [13] have provided insights into the influence of the various colloidal forces on the microstructure and the effect of this microstructure on suspension rheology. Non-dimensional structural parameter has been introduced to predict the rheology of aggregated sediment suspension [14] and semi-solid slurries [15].

Based on simple physical assumptions, we propose in this paper an original computer simulation of the microstructure of a semi-solid metallic alloy submitted to shear. First, we discuss the parameters governing the structure, then we use a simple approach to relate the microstructure with rheological properties.

\section{Structure and viscosity of sheared suspension}

The role of the solid fraction, $\Phi$, in the rheology of sheared suspensions has been extensively studied (see for instance the review of Rutgers [16]). The most used law for the $\Phi$ dependence of the viscosity is the Krieger phenomenological law. It has the advantage of pointing out the concept of maximum packing fraction $\phi_{M}$.

The role of the shear rate is less clear. We know that it tends to break-up the clusters: depending on the applied shear rate and the solid fraction. The possible structures can be schematically grouped in four different classes (see figure 2):

- At low solid fraction $\left(\Phi \leq \Phi_{M}\right)$, the structure depends on the shear rate. At low shear rate (1), very little break-up occurs, leading to a 3D interconnected network. At high shear rate (2), the break-up process dominates leaving the system as a dispersed suspension (individual particles are well separated). In between those two domains (3), the aggregation and break-up processes counterbalance, leading to a suspension of clusters with $\dot{\gamma}$ dependent characteristic size and shape.

- At high solid fraction $\left(\Phi \geq \Phi_{M}\right.$ ), close-packing is observed for any applied shear rate. The shear is then localized in particular planes.

The frontiers delimiting the different domains in figure 2 should be seen more as transition zones than strict boundaries.

In the dispersed suspension region the viscosity can be accurately described with a model developed for suspensions of hard spheres (see equation 1). In the 3D interconnected network region the deformation mechanisms are totally different involving much more the solid than the liquid. Pseudo plastic models leading to a power law, $\eta=m \dot{\gamma}^{n-1}$, describes successfully the rheology of the two-phased material (see reference 17]) .

In the intermediate region, Quemada [3] introduced the concept of Effective Volume Fraction (EVF, $\Phi_{\text {eff }}$ in equation 2) to replace the solid fraction $\Phi$ of equation 1, turning it into:

$$
\eta=\eta_{0}\left(1-\frac{\Phi_{e f f}}{\Phi_{M}}\right)^{-2.5 \Phi_{M}}
$$


The EVF takes into account the entrapped liquid not involved in the hydrodynamic

flow. $\Phi_{\text {eff }}$ is then the sum of the real solid fraction $\Phi$ and the part of the liquid which does no longer take part in the liquid flow. It could be, for example the entrapped liquid in the middle of a solid aggregate. Note that liquid does not need to be completely surrounded by solid to be entrapped (see section 4). On this basis the structure of the suspension is re-injected into equation 1 through its correlation with the EVF. For a suspension of compact clusters, the EVF would be equal to the solid fraction, while for a more open structure (containing more entrapped liquid) it could be much higher than $\Phi$ leading to higher values of viscosity. The maximum packing fraction $\Phi_{M}$ characterizes a geometrical compactness. It is a function of the size and shape distribution of the individual solid particles and does not depend on $\dot{\gamma}$. The EVF takes into account the dependence of the microstructure as a function of the applied shear rate. The structure could have been introduced in a shear dependent packing fraction $\Phi_{M}$ [18], instead of $\Phi$. Both approaches lead to the same results.

Ito and Flemings [8] used this concept to interpret experimental results on semi-solid slurries. After a time consuming micrography analysis on many planes, they managed to give a 3D reconstruction of clusters resulting from stirring at steady state. They gave an estimation of the entrapped liquid fraction and plotted the measured viscosity versus EVF for slurries solidified under different shear rates. All the experimental points were scaled back on a unique master curve.

The critical point of this analysis is the estimation of the structure dependent EVF, as a function of the shear rate. In the following section, we propose an approach predicting the structure evolution of a semi-solid slurry submitted to shear.

\section{The steady state of sheared suspension: competi- tion between aggregation and break-up}

When a semi-solid slurry is left at rest, two different mechanisms occurs:

- Oswald ripening [19] and spheroidization tend to minimize the solid/liquid surface energy by narrowing the size distribution of aggregates, and smoothing the solid particles surfaces. These phenomena are diffusion limited with a characteristic length of the order of the individual particles radius $a_{0}$. A characteristic diffusion time could be approximated by: $a_{0}^{2} / D \approx 2 \mathrm{~s}$, where $D \approx 5 \times 10^{-9} \mathrm{~m}^{2} \mathrm{~s}^{-1}$ is the diffusion coefficient of the solute in the liquid and $a_{0} \approx 100 \mu \mathrm{m}$.

- Particles aggregation occurs to lower the liquid/solid surface energy. The driving force of aggregation is the difference between twice the solid/liquid surface energy and the solid/solid surface energy: $\Delta \sigma=2 \sigma_{S L}-\sigma_{b}$. The aggregation kinetic is limited by the collision frequency which is estimated in 20 for a sheared suspension of hard spheres as: $f_{c}=(8 / \pi) \times \Phi \dot{\gamma}$. In the intermediate region of figure 2 , the characteristic time between two collisions $\left(1 / f_{c}\right)$ ranges from 1 s to 1 ms when $\dot{\gamma}$ ranges from 10 to $1000 \mathrm{~s}^{-1}$.

In the present paper, we are interested in a shear rate domain where the kinetic of aggregation is much more rapid than the kinetic of Oswald ripening (see above). We will then neglect Oswald ripening. Indeed, in a relevant time for diffusion limited mechanisms, aggregation induces more drastic changes in the microstructure than Oswald ripening. 
The aggregation mechanism is collision induced. We are interested in the physical mechanisms taking place during the contact between two particles. Two spherical objects of radius $a_{0}$ aiming at one another in a shear flow with shear rate $\dot{\gamma}$ will meet during a mean contact time $\tau_{c}$ approximated by Adler [21] for the limit of small solid fraction:

$$
\tau_{c}=\frac{5}{2} \dot{\gamma}^{-1}
$$

During the contact, if a favorable crystallographic orientation is encountered, a rigid neck will be built between the two particles (see figure 3). We now use a result derived in reference [15] concerning the growth rate of a neck between two solid spherical particles of radius $a_{0}$ (see figure 3). If $x$ is the neck radius and $\tau_{f}$ the neck formation time after the particles collision, $\tau_{f}$ follows:

$$
\tau_{f}=\frac{1}{5} A\left(\frac{x}{a_{0}}\right)^{5} a_{0}^{3}
$$

where $A$ is a constant dependent on thermo-physical properties of the material (see reference [11] for more details). Assuming the neck builds up during the contact time $\tau_{c}$, we have $\tau_{c}=\tau_{f}$, leading to an estimation of the neck size:

$$
x=\left(\frac{25}{2 A}\right)^{\frac{1}{5}} \dot{\gamma}^{-\frac{1}{5}} a_{0}^{\frac{2}{5}}
$$

The shear will tend to break the aggregates during collision. As collision of two clusters of size $\Delta L$ occurs their mean relative velocity is $\Delta L / 2 \times \dot{\gamma}$. Thus, the kinetic energy $E_{c}$ available for rupture is:

$$
E_{c}=\frac{1}{2} \rho V\left(\dot{\gamma} \frac{\Delta L}{2}\right)^{2}
$$

$\rho, V, \dot{\gamma}$ and $\Delta L$ are the mass density, the volume, the shear rate and the size of the cluster in the normal direction of the rupture plane, respectively. $E_{c}$ has to be compared with the energetic cost of the plastic rupture (classically considered to be 1000 times larger than the fragile rupture energy [22]):

$$
E_{r}=n \times \pi x^{2} \times 1000 \Delta \sigma
$$

$n$, and $\Delta \sigma$ being the number of broken necks of size $x$ (defined in equation 5) in the fracture plane, and the energy cost due to liquid / solid surface creation, respectively.

If $\dot{\gamma}$ is small, the contact time $\tau_{c}$ is long and necks will be large. Moreover, kinetic energy will be low preventing from any break-up of the resulting cluster. This situation will lead to the formation of a rigid 3D network. For large $\dot{\gamma}$ the contact time is short and the kinetic energy is large enough to prevent any aggregation. This leads to a dispersed suspension. As both aggregation and break-up are collision induced, the collision frequency influences only the kinetic of aggregation / break-up, but it does not influence the equilibrium between these two mechanisms.

In the present study, we deal with "liquid driven deformation". This means that the shear rate is sufficiently high to allow the competition between aggregation and break-up, the deformation being allowed by the free liquid between the clusters.

From these simple considerations the probabilities of aggregation and break-up between particles and clusters of particles will be derived and used to simulate cluster dynamics. 


\section{Lattice model simulation of cluster structure}

In a real system containing $\approx 10^{5}$ particles, one would have to treat $\approx 10^{3}$ clusters in order to be representative of the distribution. This is untractable with standard computer power. We are interested here in steady state interaction, which means a dynamical equilibrium between cluster aggregation and cluster fracture. We assume a sort of "ergodic" hypothesis, namely that in steady state, the size distribution of a population of clusters at a given time, is equivalent with the size distribution of a given cluster along time. Our main simplifying approach based on the above hypothesis is in considering only one representative cluster that can either aggregate with its copy, or can be broken in a random plane. The time evolution of this cluster will approximate the ensemble properties of the equilibrium semi-solid slurry.

The cluster is made up of a cubic arrangement of connected spheres (elementary particles of radius $a_{0}$ ). It is stored in a 3D matrix (cubic lattice), where 0 stands for free liquid, 1 stands for solid and 2 stands for entrapped liquid. The size of the matrix unit cell $\left(2 a_{0}\right)$ is chosen so that the elementary particle is inscribed into this cubic unit cell.

\section{Aggregation}

The aggregation probability $P_{a}$ per collision is assumed to be the probability $q_{e}$ for two particles to encounter with a favorable crystallographic orientation. As in reference [15], we consider that only low angle grain boundary $(\leq 0.25 \mathrm{rad})$ give rise to a non wetted grain boundary, leading to: $q_{e}=0.02$. This condition is equivalent to:

$$
P_{a}=q_{e}
$$

\section{Break-up}

The rupture probability per collision $P_{r}$ should be a monotonous increasing function of the ratio of the available kinetic energy over the energetic cost of the rupture $\left(E_{c} / E_{r}\right)$. At zero kinetic energy it should be zero, and for $E_{c}=E_{r}$ it should converge to 1 . The most simple form satisfying the above criterion is the simple $P_{r}=E_{c} / E_{r}$ approximation. A fracture plane is randomly chosen. The differential kinetic energy introduced in [11] is given by equation 6. The volume $V$ of the cluster is taken as $(e+s)\left(2 a_{0}\right)^{3}$ with $e$ and $s$ being the number of entrapped liquid voxels and the number of solid particles in the cluster, respectively. The energetic cost of the rupture is given by equation 7 . We then have the rupture probability per collision:

$$
P_{r}=\frac{E_{c}}{E_{r}}=\frac{2}{125} \frac{\rho a_{0}^{\frac{21}{5}}}{\pi \Delta \sigma}\left(\frac{25}{2 A}\right)^{-\frac{2}{5}} \times \frac{(e+s)\left(\frac{\Delta L}{4 a_{0}}\right)^{2} \dot{\gamma}^{\frac{12}{5}}}{n}
$$

The first group of terms in 9 depends on material properties $(\rho, \Delta \sigma, A)$ and the initial state $\left(a_{0}\right)$, whereas the second member is calculated at each step of the simulation.

During cluster/cluster collisions, aggregation or rupture may occur. However, most of the time, neither aggregation nor break-up occur and the two clusters are left as before. As we deal with the steady state there is no need to consider these collisions with no effect. The only parameter is then the ratio $P_{a} / P_{r}$. 


$$
\begin{aligned}
\frac{P_{a}}{P_{r}} & =K \times \frac{n}{(e+s)\left(\frac{\Delta L}{4 a_{0}}\right)^{2} \dot{\gamma}^{\frac{12}{5}}} \\
K & =\frac{125 \pi}{2} \frac{q_{e} \Delta \sigma}{\rho a_{0}^{\frac{21}{5}}}\left(\frac{25}{2 A}\right)^{\frac{2}{5}}
\end{aligned}
$$

Through equation 10, $K$ incorporates the material properties and the initial state. For an Al-6.5wt\%Si alloy with a $50 \mu \mathrm{m}$ globular structure, $K=5 \times 10^{8} \mathrm{~s}^{-12 / 5}$ (see Table [1).

For each step, the one-cluster algorithm randomly selects a rupture plane and calculates the break-up probability (aggregation probability is fixed to $q_{e}$ ). A number is randomly generated between 0 and $P_{a}+P_{r}$ :

- If it ranges between 0 and $P_{r}$ fracture is processed by deleting all the solid particles belonging to the rupture plane. One of the resulting clusters is randomly selected and stored in the matrix $M$.

- Else, the cluster is duplicated in a matrix $N$, randomly rotated, and the two matrices, $M$ and $N$, are then aggregated allowing interpenetration. The resulting cluster is stored in the matrix $M$.

Aggregation and rupture mechanisms are illustrated in figure 1 and a simplified algorithm of the numerical simulation is shown in figure 5 .

The number of solid particles $s$, the number of entrapped liquid voxels $e$ and the gyration radius $R$ are extracted from the matrix $M$, leading to the calculation of the effective volume fraction. The gyration radius is defined as the mean distance between the cluster center of mass and all its solid particles. Averages $(\bar{R}, \bar{s}, \bar{e})$ are calculated over the total step number. Since the mean "life-time" between two collisions is assumed to be independent of the cluster radius, there is no need to weight the averages.

One of the crucial point of this simulation is the evaluation of the entrapped liquid in the cluster. Indeed, this effect governs the effective volume fraction $\Phi_{\text {eff }}$ that will be introduced in equation 2 to predict the viscosity. It is calculated at each step and defined as follows: a liquid voxel is entrapped if 4 or more of its 6 principal directions (cubic lattice)

$(\overrightarrow{O x},-\overrightarrow{O x}, \overrightarrow{O y},-\overrightarrow{O y}, \overrightarrow{O z},-\overrightarrow{O z})$ hits any solid particle of the cluster. If only 3 directions out of the 6 point to a solid particle, the liquid is entrapped if the averaged distance between the considered liquid voxel and the 3 intersected solid particles is smaller than the cluster gyration radius $R$. In other terms, if $d_{1}, d_{2}$ and $d_{3}$ are the distances between the liquid voxel and the nearest solid particle in the 3 considered directions, the liquid is entrapped if $d_{1}^{2}+d_{2}^{2}+d_{3}^{2}<R^{2}$. For a better understanding, this procedure is visualized in figure 6 for the $2 \mathrm{D}$ case.

\section{Numerical validation:}

The simulation starts with a cluster constituted of one solid particle. It usually growths until it reaches a large enough size to be broken. A typical time evolution of the cluster gyration radius $R$ is shown in figure 7 for a shear rate of $500 \mathrm{~s}^{-1}$. Although the gyration radius $R$ fluctuates strongly in time, the distribution of $R$ is stable. The numerical convergence is reached when fluctuations of the distribution are less than $10 \%$. Figure 8 displays the cluster gyration radius distribution after various computation steps. Steady state is reached after $\approx 5000$ steps. 


\section{Results}

The result of the simulation will be presented as follows:

1. 3D cluster shape at different shear rates will be depicted.

2. The size distribution of clusters for different shear rates will be plotted and compared with an analytical model available in the literature.

3. The $\dot{\gamma}$ dependence of the mean radius $\bar{R}$ of the clusters will be discussed.

4. The parameter $\Phi_{\text {eff }} / \Phi$ will be computed as a function of the shear rate giving a more precise structure map than the one given in figure 2 .

5. Finally, the viscosity $\eta$ will be given as a function of the shear rate and the solid fraction.

\section{Cluster shape}

Figure 9 shows a cluster of radius $R$ with its entrapped liquid for two different shear rates. For small $\dot{\gamma}$, aggregation is dominant leading to a more open structure entrapping more liquid.

\section{Cluster size distribution}

Figure 10 exhibits normalized radius distributions for different shear rates. Size distribution of particles can be accurately described by a "log-normal" law, frequently encountered in fragmentation problems (see reference 23]). Since these experiments are ensemble distributions, whereas our simulation deals with time evolution, this similarity gives some confidence into the used "ergodic"-type hypothesis.

The normalization brings the simulated distribution for various shear rates to a single master curve. The size dispersion is then proportional to the mean size of the clusters: lower is the shear rate, larger is the mean cluster radius and larger is the size dispersion. For low shear rate, the cluster can explore either small and large sizes, whereas, for high shear rate, only small sizes are possible.

In figure 10, one can compare the simulated distribution with the mathematical model of Takajo 24 often used as a model for aggregation processes. This model based on homogeneous coalescence frequency gives a normalized size distribution at steady state. These two different approaches lead to comparable profiles, giving some confidence into the general basis of our simulation.

\section{Mean cluster radius $v s$. shear rate}

The obtained mean gyration radius values are plotted as a function of the shear rate on figure 11 The mean gyration radius $\bar{R}$ decrease with increasing shear rate following a power law $\bar{R} \propto \dot{\gamma}^{-0.7}$. This dependence is comparable with the power law estimated for the compact cluster scenario 《11]: $R \propto \dot{\gamma}^{-0.6}$. It can be observed that, above a given $\dot{\gamma}$ only elementary particles remain, hence there is no more evolution of $\bar{R}$.

As the physical parameter $K$ depends strongly on the size of the individual particles $a_{0}$ (see equation 10), computations are presented for two different reasonable values of $a_{0}$. For the smaller elementary particle size, cluster are larger at some given shear rate and solid fraction. 


\section{Effective volume fraction vs. shear rate}

Figure 12 displays the calculated ratio $\Phi_{\text {eff }} / \Phi$ as a function of $\dot{\gamma}$ for two different elementary particle sizes. For high $\dot{\gamma}$, (high enough to break all the necks), there is no entrapped liquid $\left(\Phi_{\text {eff }} / \Phi=1\right)$. For small $\dot{\gamma}$, more connected structures leads to high fraction of entrapped liquid $\left(\Phi_{\text {eff }} / \Phi \approx 2\right)$.

In figure 13 we can identify the three different domains introduced in section 2 (figure 2).

- For high $\dot{\gamma}$ values $\left(\geq \dot{\gamma}_{s}\right)$, the dispersed suspension-like behavior is observed. $\dot{\gamma}_{s}$ is reached when the mean radius of the clusters is equal to the radius of the elementary solid particles, that is to say when the effective volume fraction is equal to $\Phi$. Note that an approximation of $\dot{\gamma}_{s}$ can be obtained equaling $P_{a}$ and $P_{r}$ of equation 10. We find for Al-6.5wt\%Si with initial particles size $a_{0}=100 \mu \mathrm{m}: \dot{\gamma}_{s}=1300 \mathrm{~s}^{-1}$. This value is clearly in accordance with the transition domain of figure 12 .

- For low $\dot{\gamma}$ values $\left(\leq \dot{\gamma}_{n}\right)$ a rigid network sets in. The estimation of $\dot{\gamma}_{n}$ depends on the gelation mechanism:

- experimentally, the apparent rigid network will appear when the mean radius of the clusters reaches the characteristic size of the measurement apparatus (Couette Rheometer for example).

- in theory, the apparent rigid network will appear when $E V F=\Phi_{M}$ : the whole volume of the sample is invaded by a unique cluster.

$\dot{\gamma}_{n}$ is found to be $\Phi$ dependent. We can observe that the simulation leads to a more realistic description of the structure(figure 13) than in figure 2. Indeed, lower is the solid fraction, higher is the liquid fraction involved in the hydrodynamic flow for some given $\dot{\gamma}$ and aggregation state characterized by $e$ and $s$.

- In the intermediate range we have a suspension of clusters with decreasing size and entrapped liquid fraction as $\dot{\gamma}$ increase.

\section{Viscosity vs. shear rate}

For the calculation of the viscosity, the entrapped liquid is taken into account trough (a) the mean number of entrapped liquid voxels $\bar{e}$, and (b) the amount of entrapped liquid within a cubic voxel containing a solid sphere, which depends on the mean number $n_{n}$ of solid neighbors of a particle over the 26 possible neighbors in the cubic lattice. The liquid fraction within a unit cell is 0.42 (difference of volume between the cube and its inscribed sphere). The viscosity follows:

$$
\begin{aligned}
& \eta=\eta_{0}\left(1-\frac{\Phi_{\text {eff }}}{\Phi_{M}}\right)^{-2.5 \Phi_{M}} \\
& \Phi_{e f f}=\Phi\left(1+\frac{\bar{e}}{\bar{s}}+\frac{(0.42) \frac{n_{n}}{26}}{s}\right)
\end{aligned}
$$

where $\bar{e}$ and $\bar{s}$ are the mean number of entrapped liquid sites and the mean number of solid sites in the cluster, respectively. With a suspension of hard spheres, $\Phi_{M}$ would have been 0.65 , but as we deal with polydisperse and deformable solid particles, it is chosen to be 1 . Indeed, the liquid phase has been proven to remain connected for solid fraction up to 0.8 . 
These equations lead to figure 14 where viscosity is plotted versus the solid fraction. The viscosity exhibits the typical profile of a semi-solid mixture [2]. The viscosity increases with the solid fraction until the solid fraction reaches a threshold value where the suspension behaves more like a solid than a liquid. Higher is the shear rate, higher is this threshold value.

\section{Comparison with real microstructure and experi- mental viscosity measurement}

\subsection{D microstructure visualization}

The advantage of such a simulation is the ability of viewing 3D structures. It is of interest to compare them with real structures resulting from serial cutting and micrography analysis made by Ito [8]. Figure 15 shows experimental and simulated 3D cluster structures. The physical parameter $K$ (see equation 10) has been calculated for the Al-6.5wt\%Si alloy with a elementary particle radius $a_{0}=50 \mu \mathrm{m}$. Note that the cluster exhibited by Ito is not representative of the population. Thus, we extracted from the simulation a cluster with comparable size.

\subsection{Viscosity measurement}

Comparison with experimental viscosity measurement is not an easy task. Viscosity measurements are usually performed after solidification under shear rate [8]. Both the solidification and the aggregation processes takes place simultaneously.

In our approach a suspension of dispersed individual globular particles is submitted to shear until it reaches its steady state of aggregation. This procedure decorrelates solidification and aggregation. However, the solidification process is assumed to have a slighter effect than the shear on both the structure and the viscosity. We then compare the simulation results with experimental viscosity measurements performed on Al-6.5wt\% Si alloys partially solidified under stirring [8]. Those experimental results were obtained with Couette rheometer and the measurements were performed at steady state: when the shear stress is stable over time. This experimental procedure allows us to consider that the aggregation degree of the microstructure corresponds to the steady aggregation degree. The good agreement between simulation results and experimental results plotted in figure 16 tends to validate both the former assumption and the basis of the simulation.

\subsection{Particle size distribution}

In figure 17, we plotted the simulated distribution master curve (see section 6) and experimental normalized size distributions resulting from microstructure analysis [19]. The master curve seem to fit accurately the real distribution profile.

\section{Conclusion}

The structure of semi-solid slurries results from the competition between aggregation due to surface forces and break-up induced by the shear field. Aggregation probability is set to be constant. Break-up probability is then set to be proportional to the ratio of the available kinetic energy over the rupture energy. Using a one-cluster algorithm, we 
supposed that the time evolution of a single cluster approximates the ensemble distribution of a population of clusters ("ergodic" hypothesis). Simulation of the time evolution of our representative cluster describes accurately the structure of the slurry with no adjustable parameter and estimates successfully the viscosity as a function of the applied shear rate and the volumic solid fraction. The present work allows a better understanding of the structure evolution of sheared semi-solid metallic alloys in the $(\Phi, \dot{\gamma})$ plane. In a future work, ripening could be also taken into account through a reinforcement of the necks along time after the particles encounter.

The present approach could be easily transferable to any type of suspension as far as the interacting forces between solid particles can be estimated.

The evaluation of the entrapped liquid fraction could be performed using a general dimension code for computing convex hulls. "QuickHulls" from the Geometry Center, University of Minnesota, USA, could provide us with a powerful tool.

\section{Acknowledgment}

We are grateful to C. Martin, M. Papoular, M. Suéry and A. Zavaliangos, for useful discussions. This work has been partly funded by Electricité De France (EDF) and CEA. One of us (Michel Perez) is supported by a MENRT grant and partial financial support from Région Rhône-Alpes is gratefully acknowledged.

\section{References}

[1] Flemings, M. C., Met. Trans. B, 1991, 22B, 269.

[2] Joly, P. A. and Mehrabian, R., J. Mat. Science, 1976, 11, 1393.

[3] Quemada, D., J. de Méc. Th. et Appl., 1985, special, 267.

[4] Quemada, D., Eur. Phys. J., 1998, AP1, 119.

[5] Barnes, H. A., J. Rheology, 1989, 33, 329.

[6] Krieger, I. M. and Dougherty, T. J., Trans. Soc. Rheol., 1959, 3, 137.

[7] Jeffrey, D. J. and Acrivos, A., AlChE Journal, 1976, 22, 417.

[8] Ito, Y., Flemings, C. and Cormie, J. A., in Nature and properties of semi-solids materials, ed. J. A. Sekhar and J. A. Dantziz. TMS, San Diego, CA, 1992.

[9] Poulain, P., Bibette, J. and Weitz, D. A., Eur. Phys. J. B, 1999, 7, 277.

[10] Buffière, J. Y., Maire, E., Lormand, P. C. G. and Fougères, R., Acta. Mater., 1999, 47, 1613.

[11] Barbé, J. C., Perez, M. and Papoular, M., J. Phys. : Cond. Matter, 2000, 12, 2567.

[12] Rastogi, S. R., Wagner, N. J. and Lustig, S. R., J. Chem. Phys., 1996, 104, 9234.

[13] Bradi, J. F. and Bossis, G., Ann. Rev. Fluid Mech., 1988, 20, 111.

[14] Toorman, E., Rheol. Acta, 1997, 36, 56. 
[15] Martin, C., Kumar, P. and Brown, S., Acta Metall., 1994, 42, 3603.

[16] Rutgers, I. R., Rheologica Acta, 1962, 2, 305.

[17] Laxmanan, V. and Flemings, C., Met. Trans. A, 1980, 11A, 1927.

[18] Wildemuth, C. R. and Williams, M. C., Rheologica Acta, 1984, 23, 627.

[19] Wan, G. and Sahm, P. R., Acta Metall., 1990, 6, 967.

[20] Mason, S. G. and Bartok, W., in Rheology of Disperse systems, C. Mill, Pergamon Press, 1957.

[21] Adler, P., Nadim, A. and Brenner, H., in Advances in Chemical Engineering, volume 15, J. Wei, Academic Press, NY, 1994.

[22] Ashby, M. F. and Jones, D. R. H., in Engineering Materials 1 : An Introduction to Their Properties and Applications, Paperback, 1996.

[23] Reed, J. S., in Principles of ceramic processes, Wiley-Interscience, 1988.

[24] Takajo, S., Kaysser, W. and Petzow, G., Acta Metall., 1984, 32, 107. 


\begin{tabular}{|c|c|}
\hline Symbol & Meaning \\
\hline$A$ & Constant depending on thermophysical parameters $\left(1.7 \times 10^{16} \mathrm{~s} \mathrm{~m}^{-3}\right)$ [11] \\
\hline$a_{0}$ & Initial particles Radius \\
\hline$D$ & Diffusion coefficient of the solute in the liquid $\left(5 \times 10^{-9} \mathrm{~m}^{2} \mathrm{~s}^{-1}\right)$ \\
\hline$d_{1}, d_{2}, d_{3}$ & $\begin{array}{l}\text { Distance between a liquid voxel and the nearest solid particle in the direc- } \\
\text { tions }(\overrightarrow{O x}),(\overrightarrow{O y}),(\overrightarrow{O z})\end{array}$ \\
\hline$e, \bar{e}$ & Number and mean number of entrapped liquid voxels in a cluster \\
\hline$E_{b}$ & Rupture energy of a cluster \\
\hline$E_{c}$ & Kinetic energy of a cluster \\
\hline$f_{c}$ & Collision frequency of clusters in a shear field \\
\hline$K$ & Physical constant defined in equation 10 \\
\hline$M, N$ & 3D Matrice used to store the cluster \\
\hline$n$ & Number of necks in the fracture plane \\
\hline$P_{r}$ & Breaking probability \\
\hline$P_{a}$ & Aggregation probability \\
\hline$q_{e}$ & $\begin{array}{l}\text { Probability for two particles to encounter with favorable crystallographic } \\
\text { orientation }\end{array}$ \\
\hline$R, \bar{R}$ & Cluster gyration radius and cluster mean gyration radius \\
\hline$s, \bar{s}$ & Number and mean number of solid particles in a cluster \\
\hline$V$ & Volume of the cluster (solid + entrapped liquid) \\
\hline$x$ & Neck radius \\
\hline$\Delta L$ & Size of the cluster in the normal direction of the rupture plane \\
\hline$\Delta \sigma$ & Surface Energy due to liquid / solid surface creation $\left(0.34 \mathrm{~J} \mathrm{~m}^{-2}\right)$ \\
\hline$\eta$ & Viscosity of the suspension \\
\hline$\eta_{0}$ & Viscosity of the liquid (20 mPa.s) [8] \\
\hline$\dot{\gamma}$ & Shear rate \\
\hline$\dot{\gamma}_{g}$ & Gelation shear rate \\
\hline$\dot{\gamma}_{s}$ & Shear rate from which no aggregation occurs \\
\hline$\Phi$ & Volumic solid fraction \\
\hline$\Phi_{\text {eff }}$ & Effective Volume Fraction (EVF) \\
\hline$\Phi_{M}$ & Maximum packing fraction \\
\hline$\rho$ & Density $\left(2350 \mathrm{~kg} \mathrm{~m}^{-3}\right)$ \\
\hline$\sigma_{S L}$ & Liquid / Solid surface energy $\left(0.17 \mathrm{~J} \mathrm{~m}^{-2}\right)$ \\
\hline$\sigma_{b}$ & $\begin{array}{l}\text { Grain boundary surface energy (negligible: Coincidence Site Lattice hy- } \\
\text { pothesis) }\end{array}$ \\
\hline$\tau_{c}$ & Contact time between two particles in a shear field \\
\hline$\tau_{f}$ & Formation time of a neck between two particles \\
\hline
\end{tabular}

Table 1: Symbols and values for Al-6.5wt\% Si 


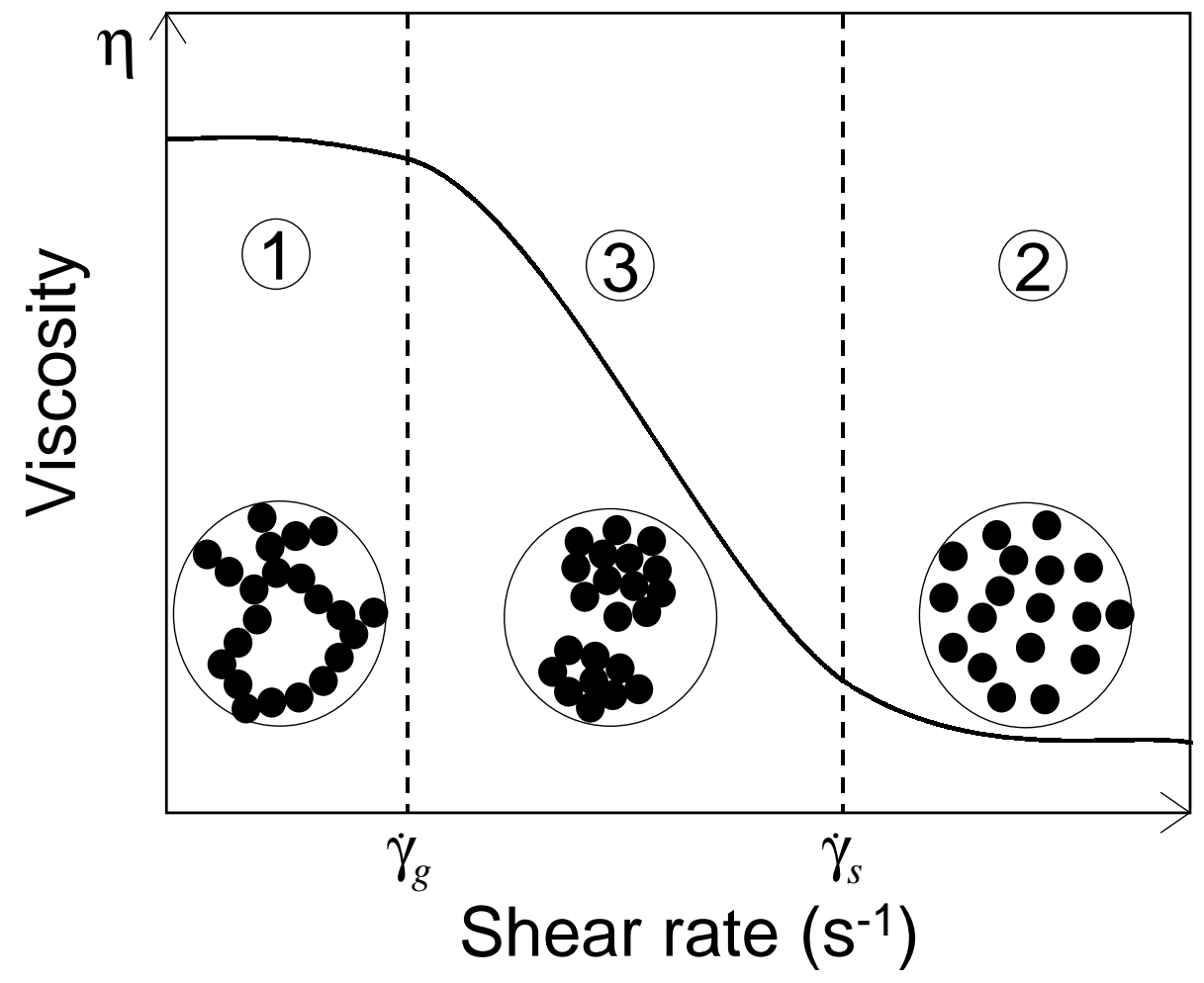

Figure 1: Rheofluidization: Viscosity decreases between the shear rates $\dot{\gamma}_{g}$ and $\dot{\gamma}_{s}$. 1Percolating network, 2- dispersed suspension, 3- suspension of clusters. 


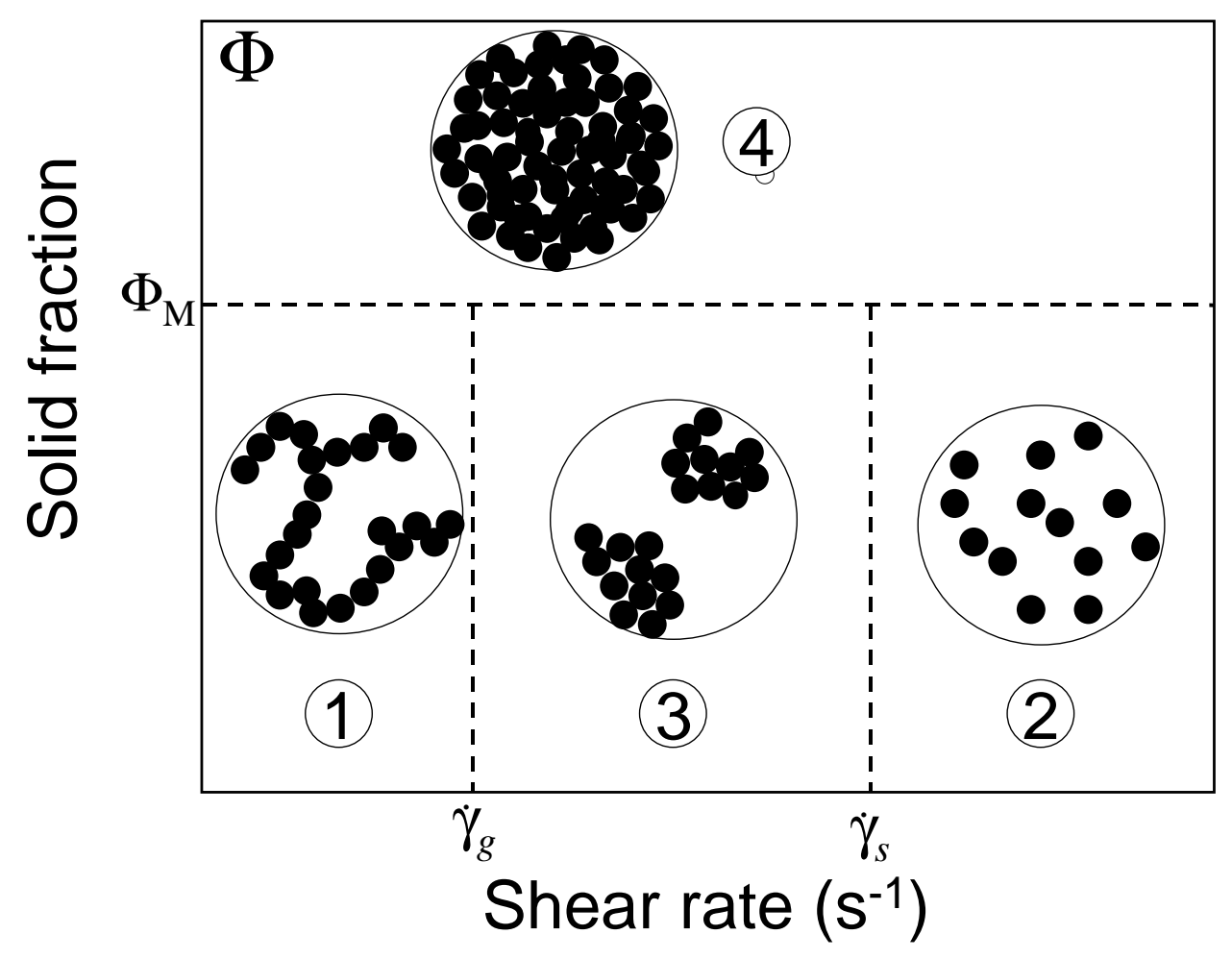

Figure 2: Structure of the suspension in the $(\dot{\gamma}, \Phi)$ phase-space. 1-Gel, 2-Suspension of individual particles, 3-Suspension of clusters, 4-Compact arrangement. In this paper we are mostly interested in regions 2 and 3 . 


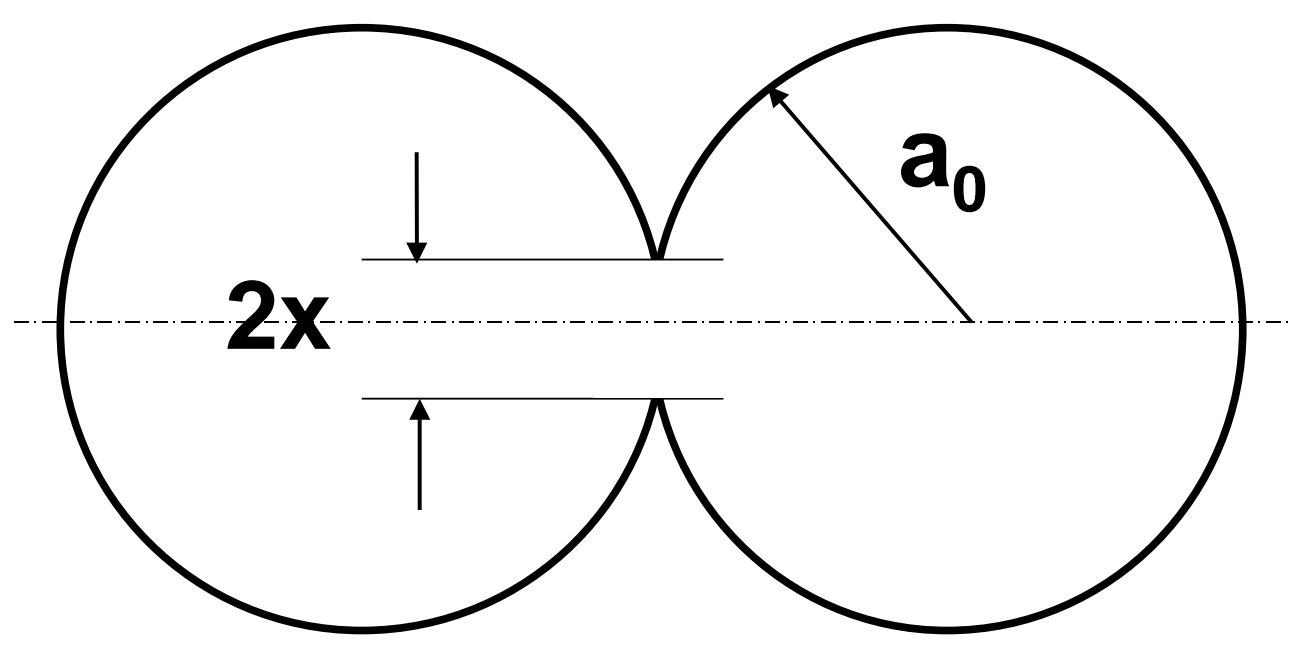

Figure 3: Neck growth between two solid particles. 

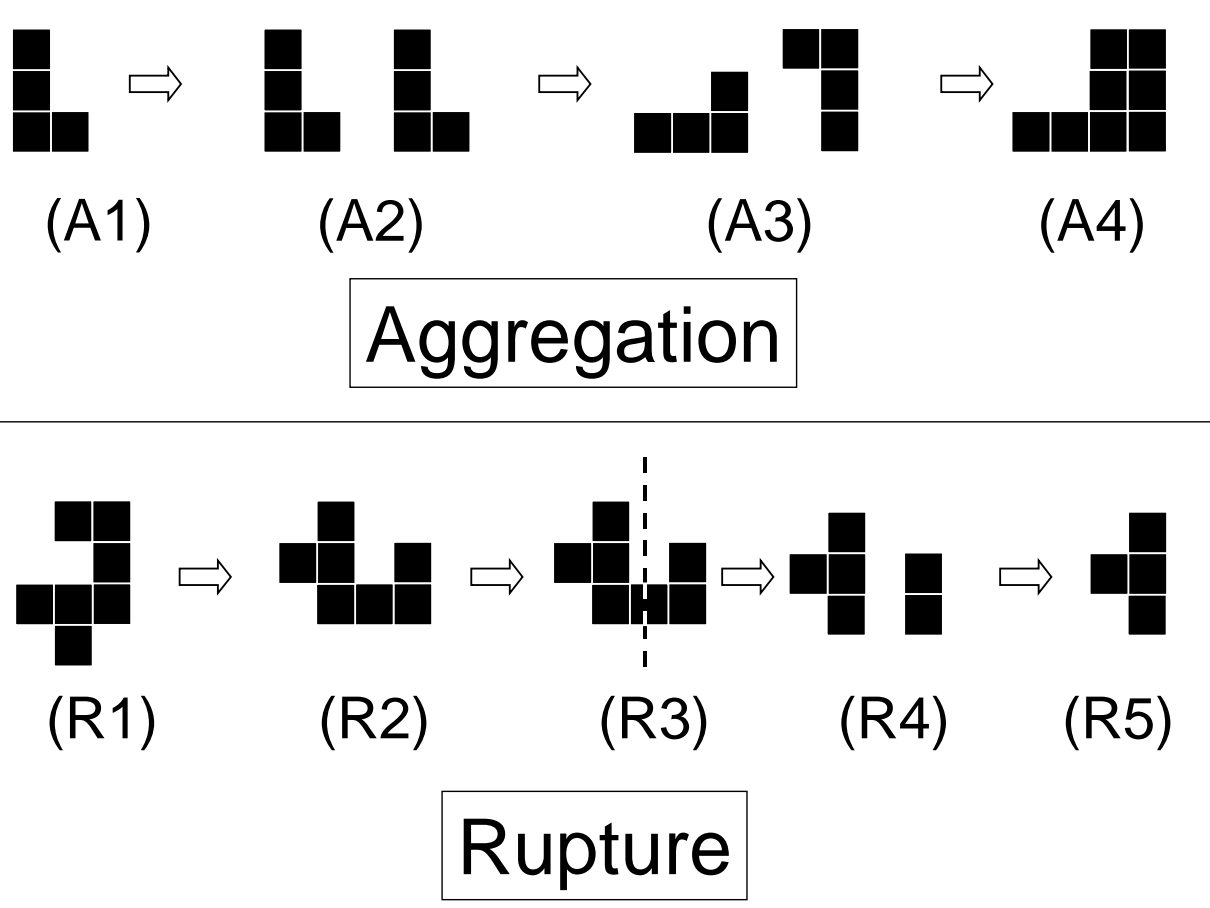

Figure 4: Aggregation process: (A1) Initial cluster - (A2) Initial cluster is duplicated (A3) Random rotation - (A4) Sticking of the two clusters. Rupture process: (R1) Initial cluster - (R2) Random rotation - (R3) Random selection of a fracture plane - (R4) Erasing the particles in the fracture plane - (R5) Random selection of the resulting cluster. Note that the fracture probability depends on the number of neck to be broken in the fracture plane. 
Randomly rotate $M$

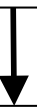

Calculate the break-up and aggregation probabilities: $P_{r}$ and $P_{a}$ and select whether breaking or sticking occurs

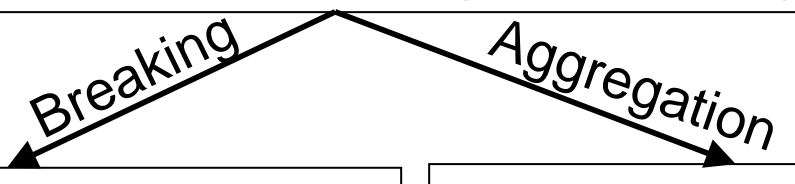

Delete the solid particles in the breaking plane

Copy $M$ to $N$ $\downarrow$ Select randomly
one of the resulting
clusters, and put it in $M$ Select randomly
one of the resulting
clusters, and put it in $M$ Select randomly
one of the resulting
clusters, and put it in $M$ and rotate $M$ and $N$
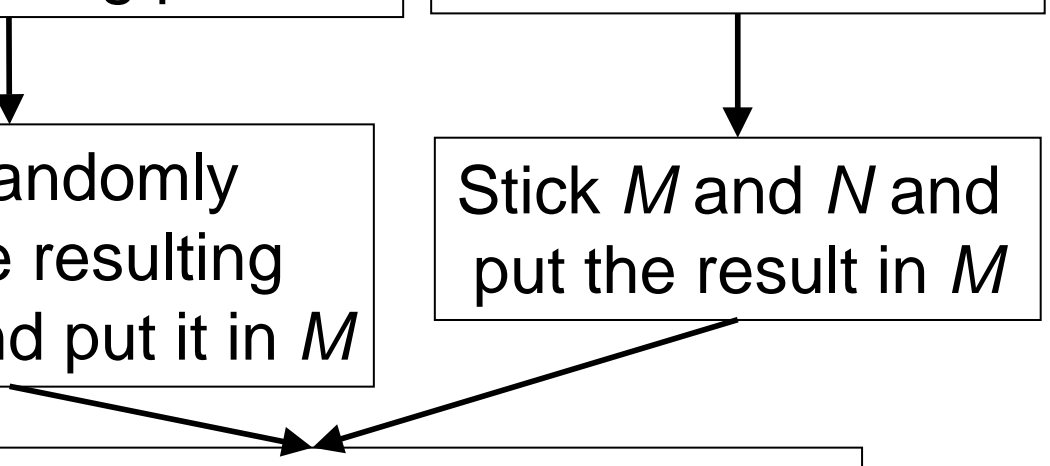

For each liquid voxel of $M$, select whether it is entrapped or not

Calculate the new:

- mean gyration radius: $R$

- mean solid particle number: $s$

- mean entrapped liquid particle number: $e$ 


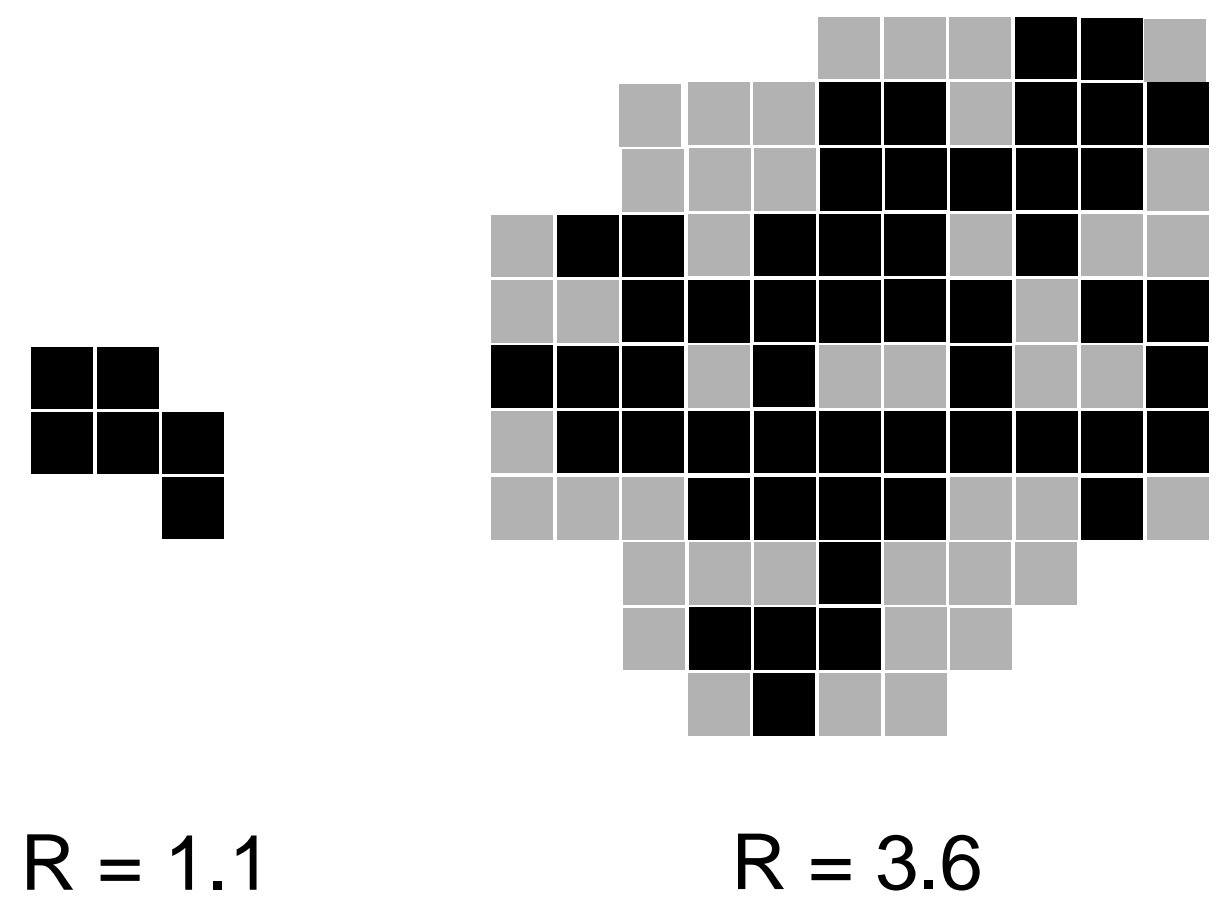

Figure 6: Representation of the entrapped liquid (grey) for a 2D Cluster: The liquid is entrapped if three or four of the principal directions point to any solid particle (black). In the case of two directions pointing to solid particles, the mean distance to solid is compared with the cluster gyration radius. 


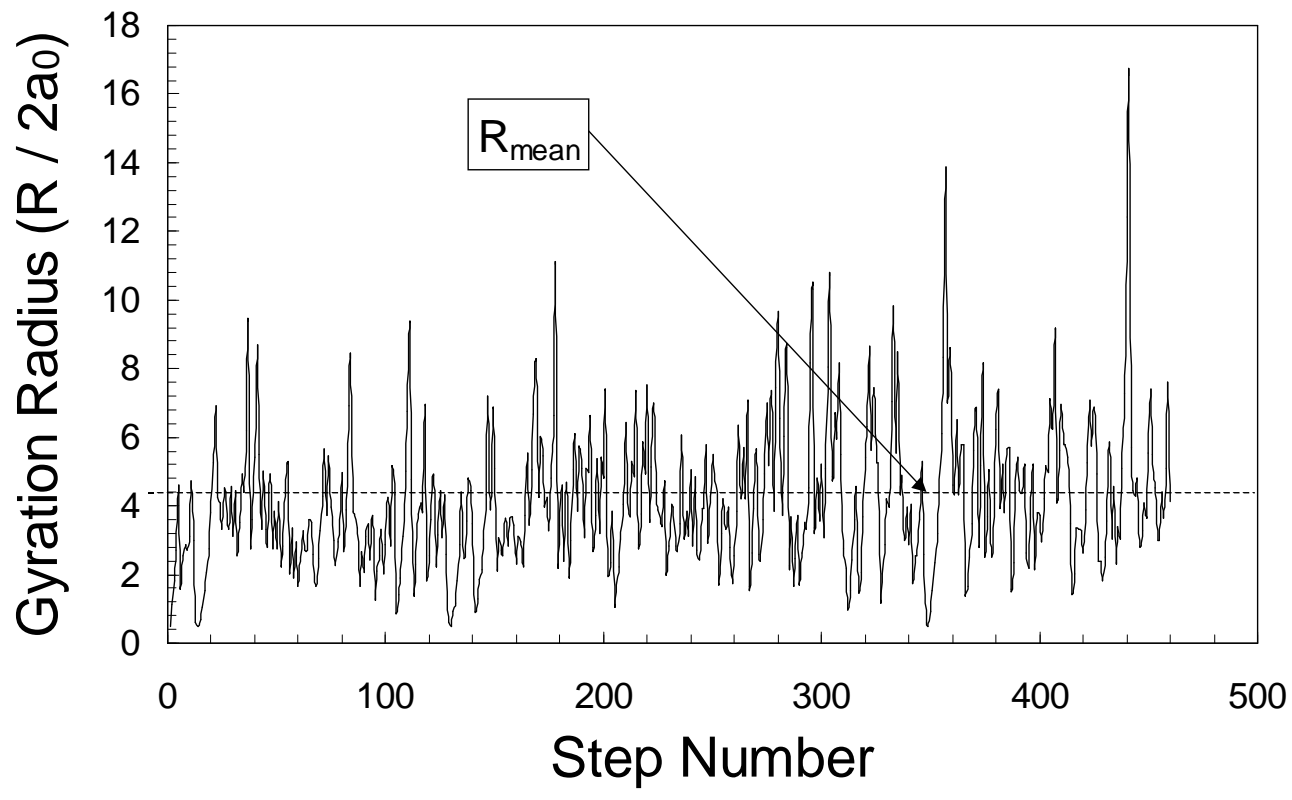

Figure 7: Time evolution of the cluster gyration radius. $R$ fluctuates a lot, but its time evolution is assumed to be representative of the spatial distribution of the clusters. 


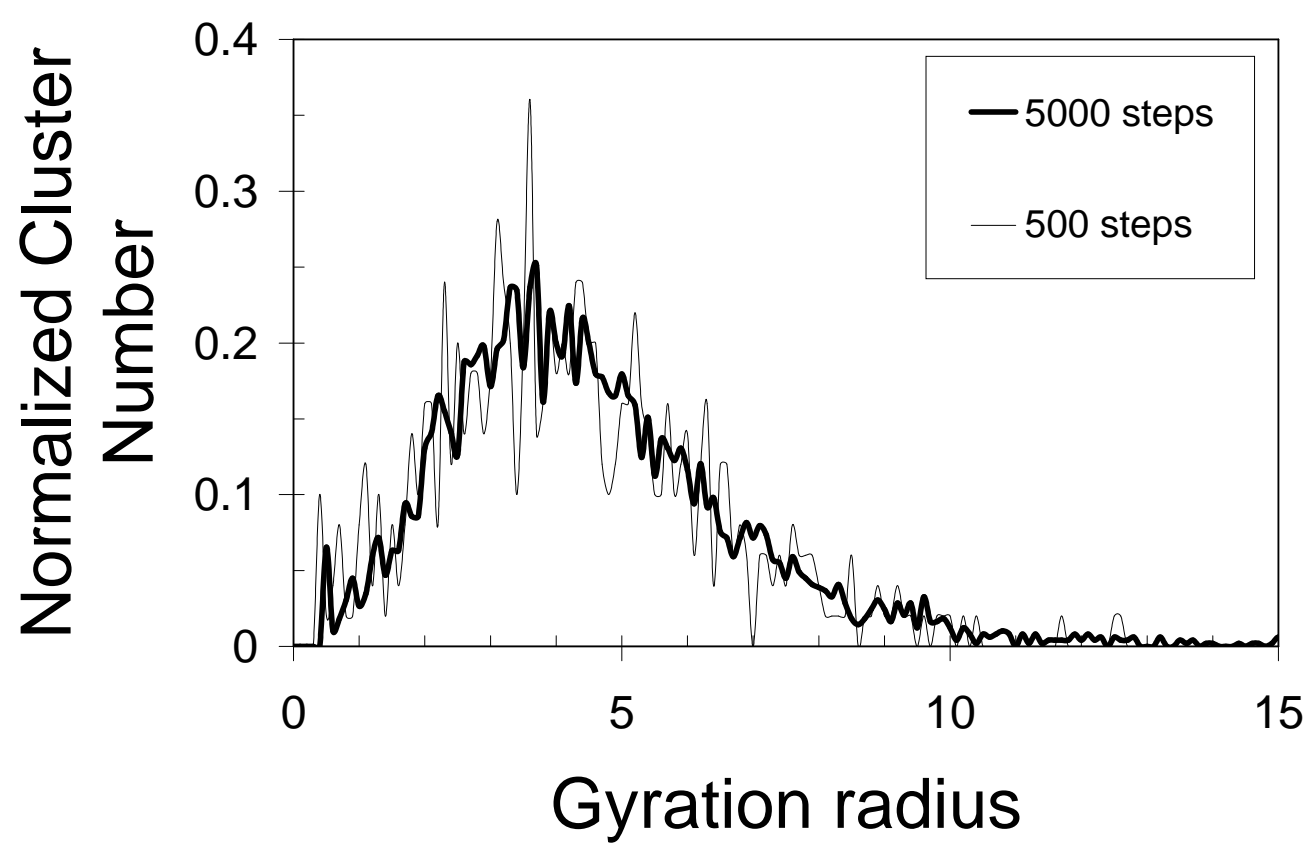

Figure 8: distribution of $R$ after various computation steps. Steady state is reached when distribution fluctuations are less than $10 \%$. 

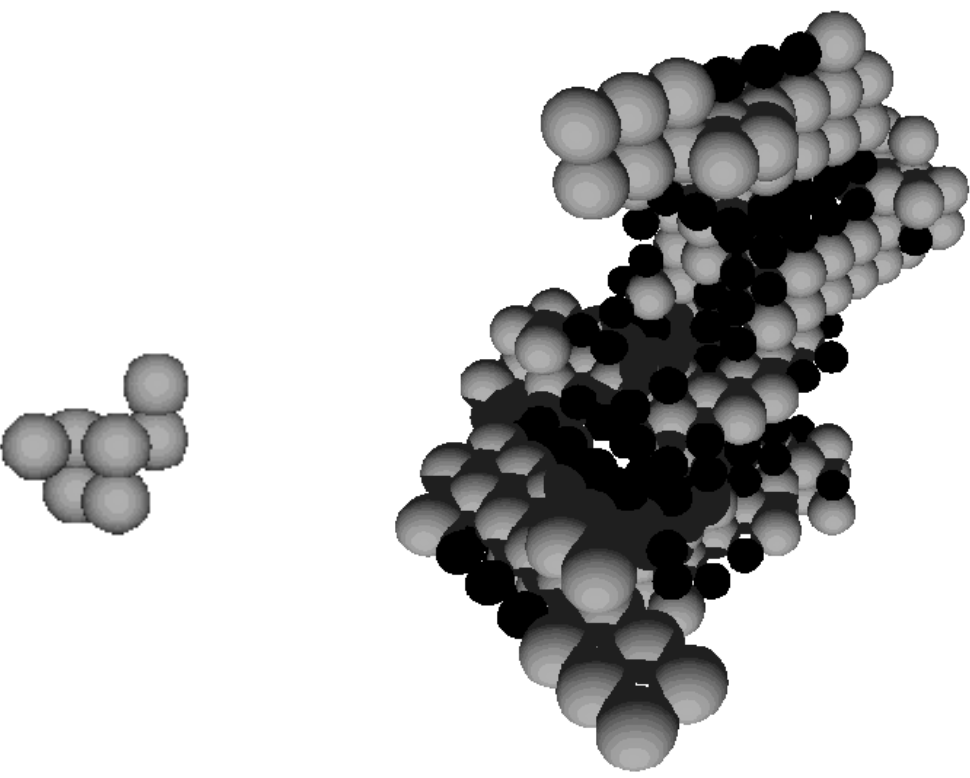

$\dot{\gamma}=500 \mathrm{~s}^{-1}$

$\dot{\gamma}=100 \mathrm{~s}^{-1}$

Figure 9: Clusters for two different shear rates. Liquid is transparent, solid is grey, and entrapped liquid is black. Larger cluster corresponds clearly to higher entrapped liquid fraction. 


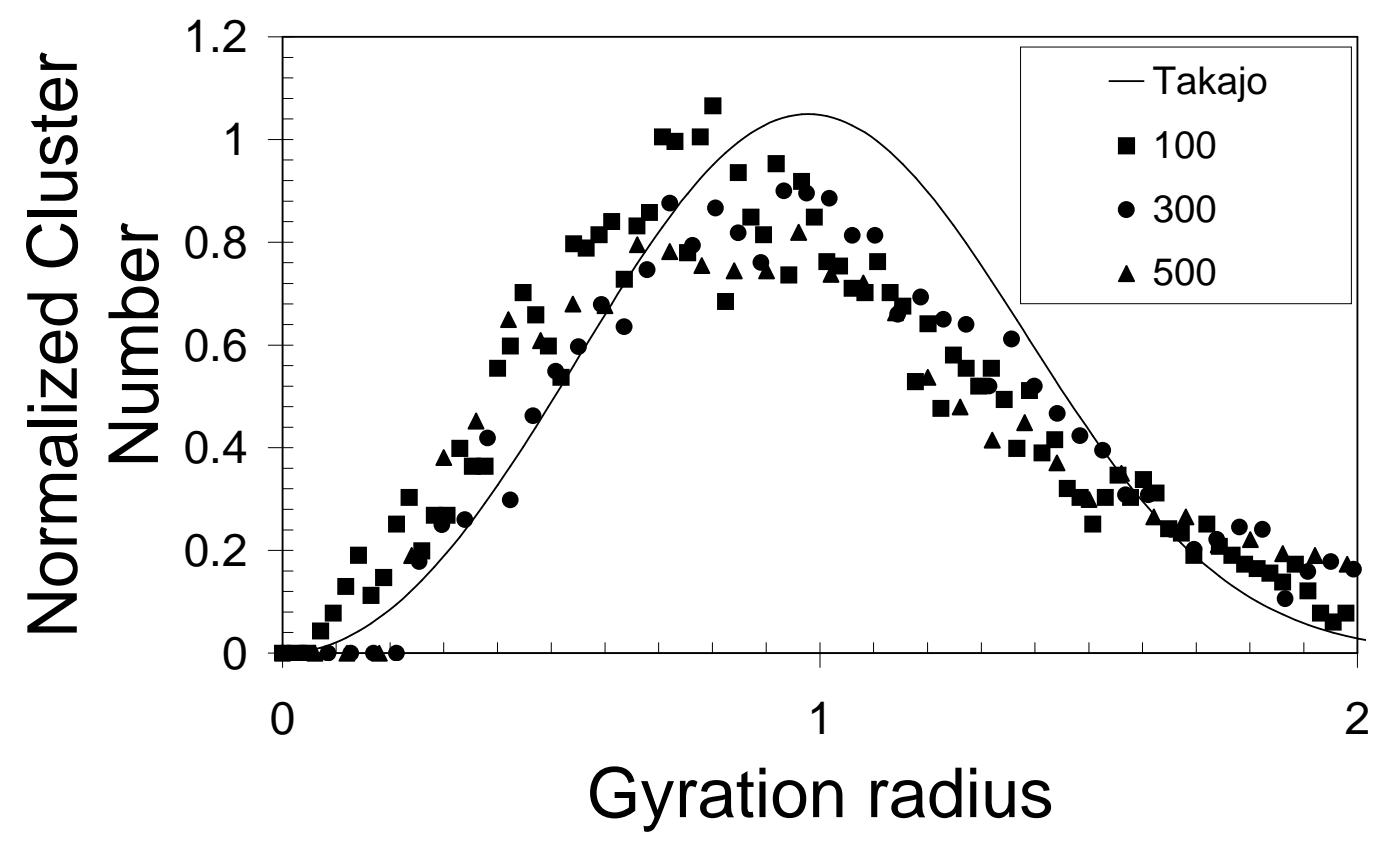

Figure 10: Gyration radius distribution for 5000 simulation steps at various shear rates compared with the mathematical model of Takajo [24] 


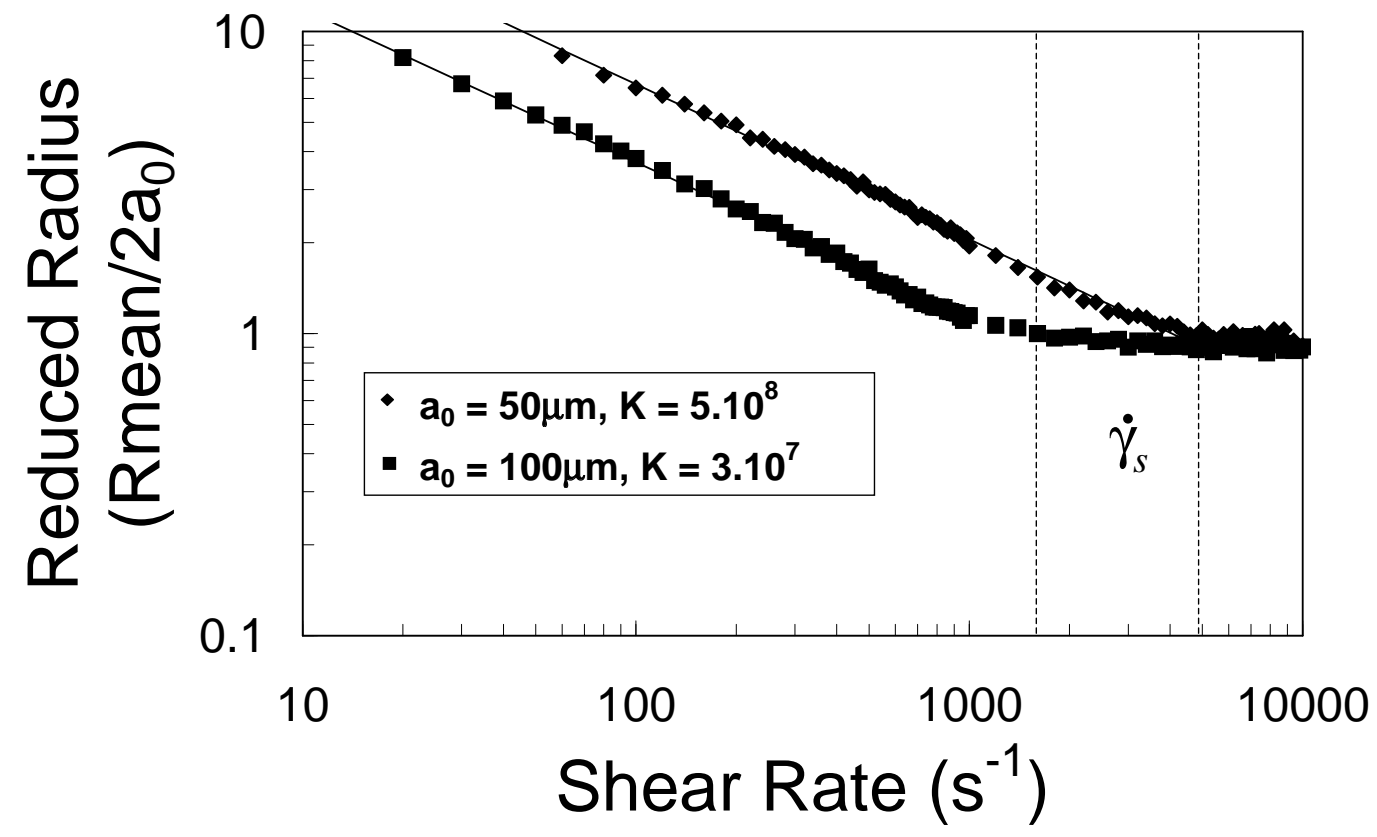

Figure 11: The $\dot{\gamma}$ dependence of the mean gyration radius. For $\dot{\gamma}$ higher than $\dot{\gamma}_{s}$, the shear breaks the clusters into individual particles. 


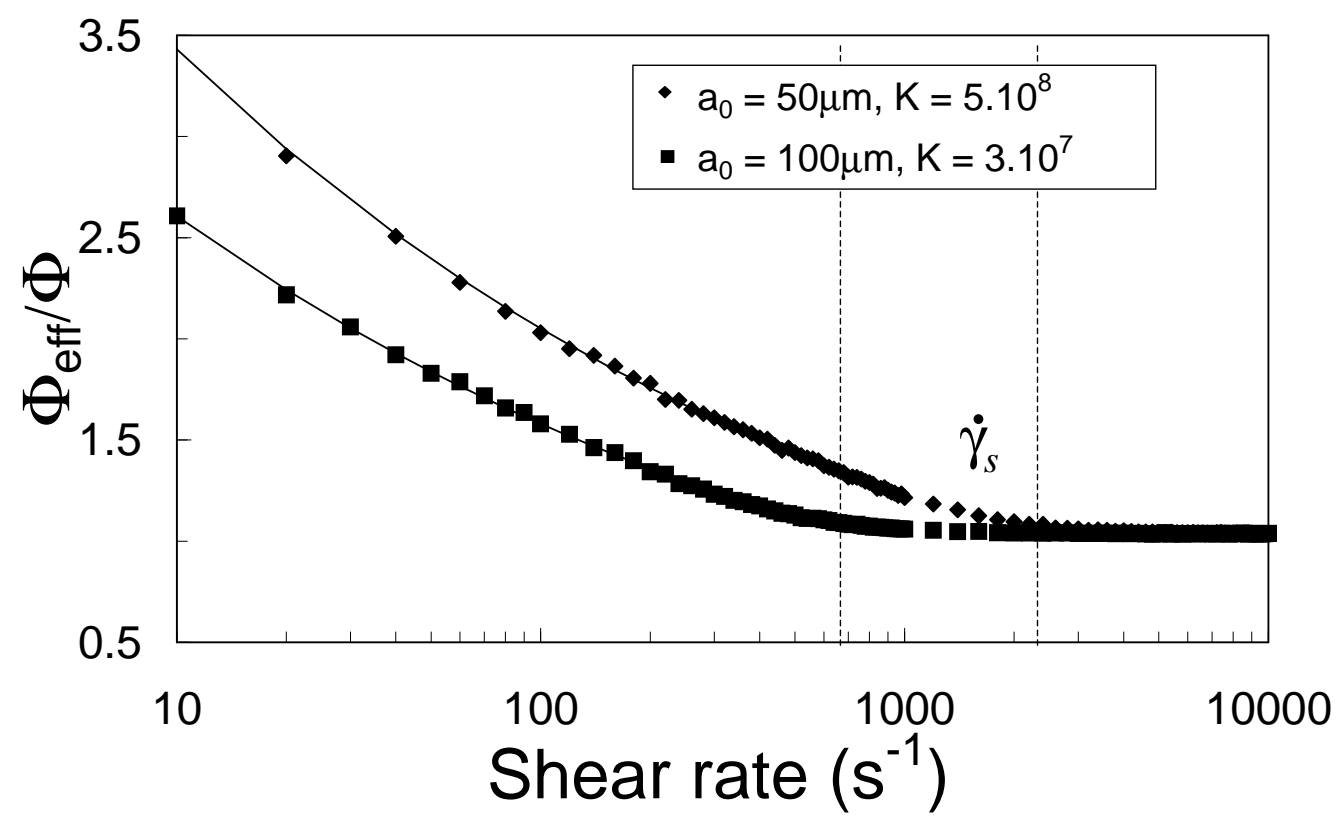

Figure 12: The ratio $\Phi_{\text {eff }} / \Phi$ as a function of $\dot{\gamma}$ for two values of the simulation constant corresponding to two initial particle sizes. For $\dot{\gamma}$ higher then $\dot{\gamma}_{s}$, the structure is too small to trap any liquid. 


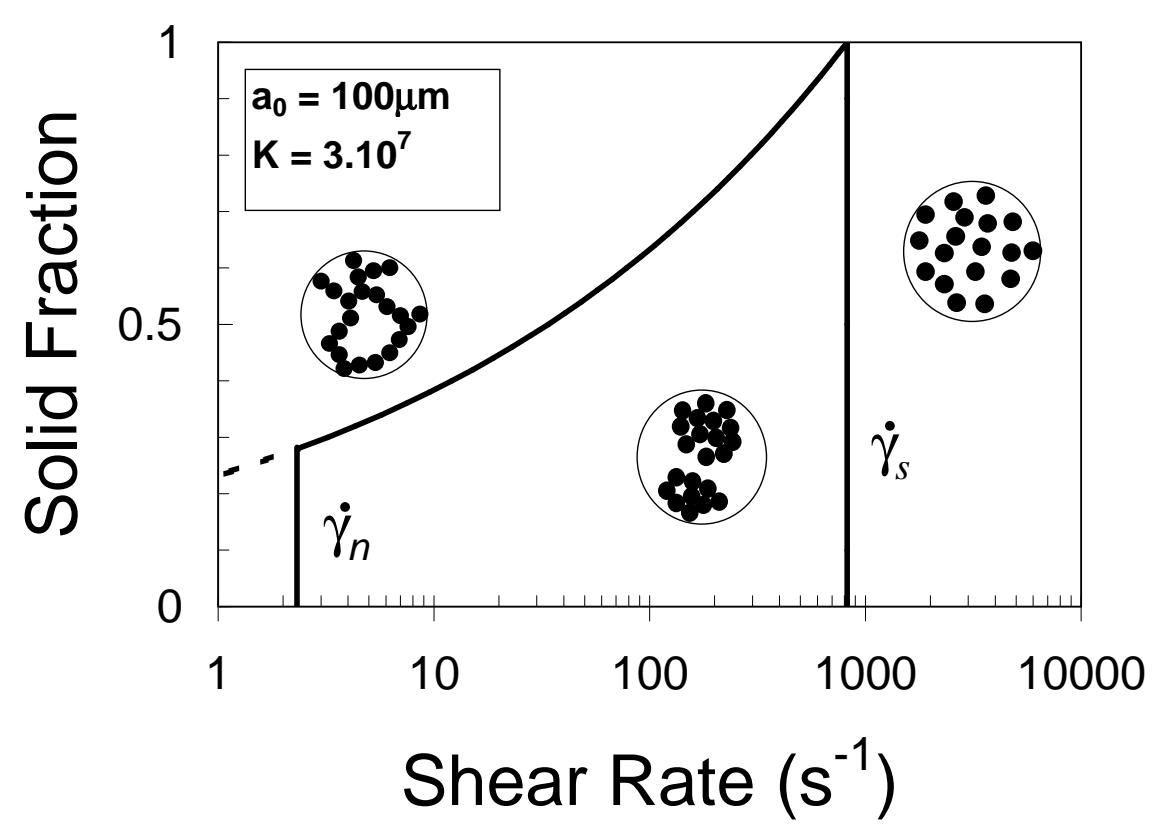

Figure 13: Suspension structure depending on $\dot{\gamma}$ and $\Phi . \dot{\gamma}_{n}$ is now $\Phi$ dependent, except for low solid fraction where gelation occurs when a cluster reach the size of the apparatus gap (left vertical line). 


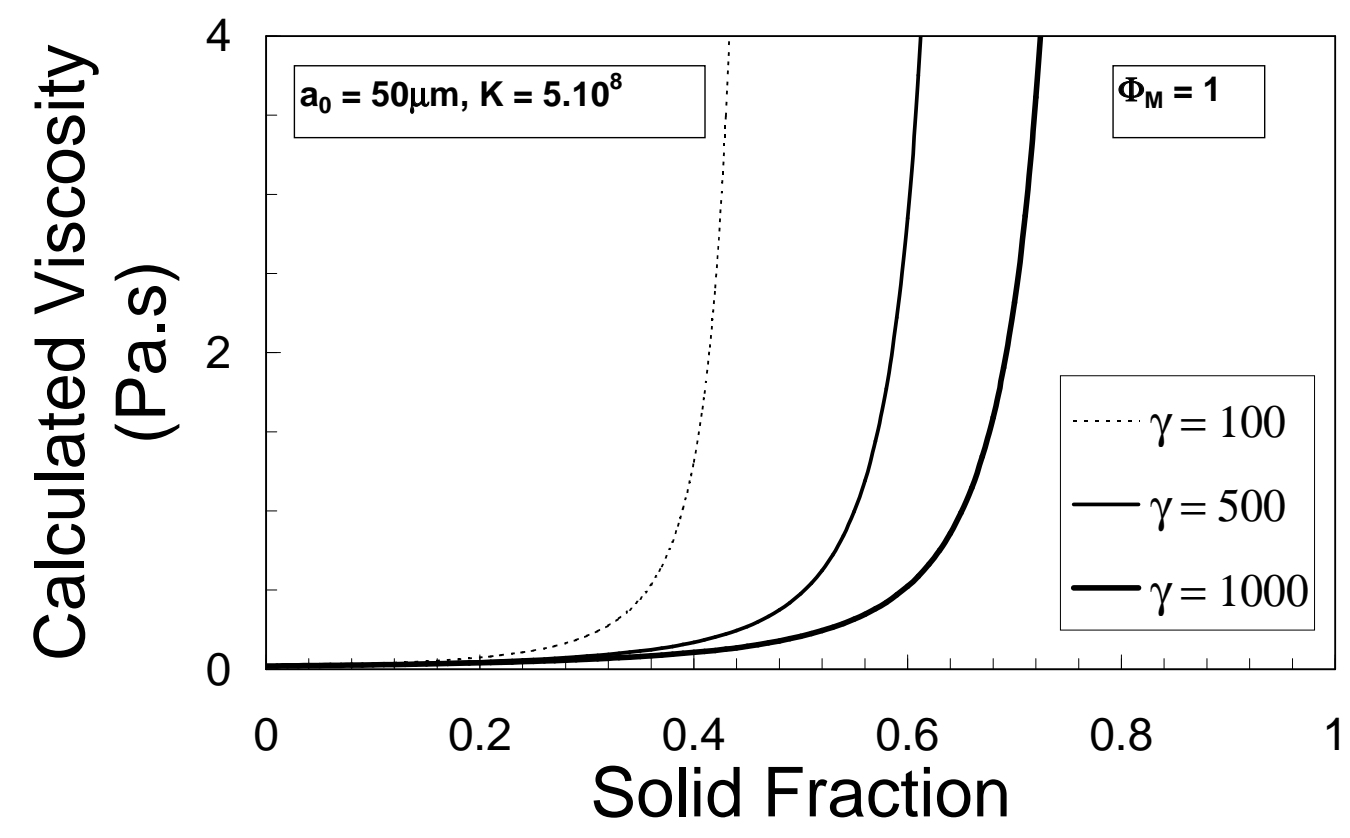

Figure 14: Viscosity as a function of the solid fraction for different shear rate. Higher is the shear rate, higher is the solid fraction leading to gellation. 

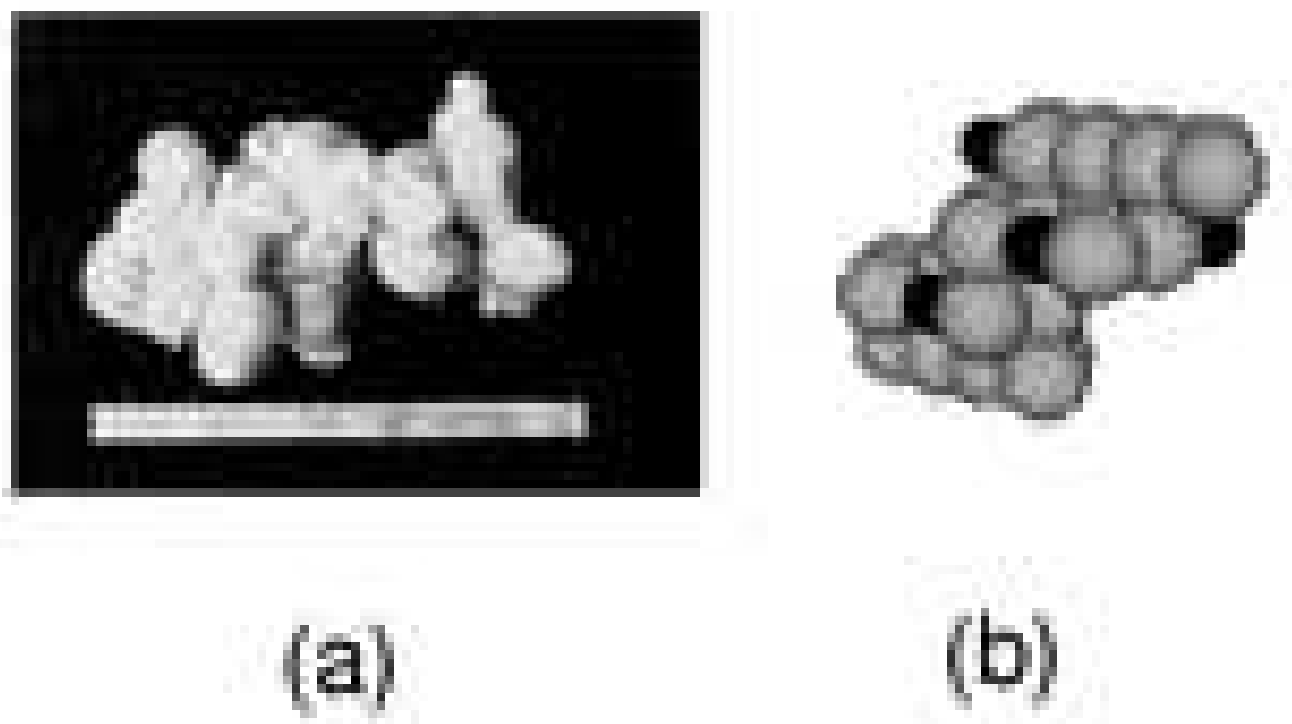

Figure 15: Experimental and simulated cluster structure taken from an Al-6.5wt\% Si semisolid slurry sheared at $900 \mathrm{~s}^{-1}$. (a) 3D reconstruction extracted from reference [8] (b) Simulated with $K=5 \times 10^{8}$ 


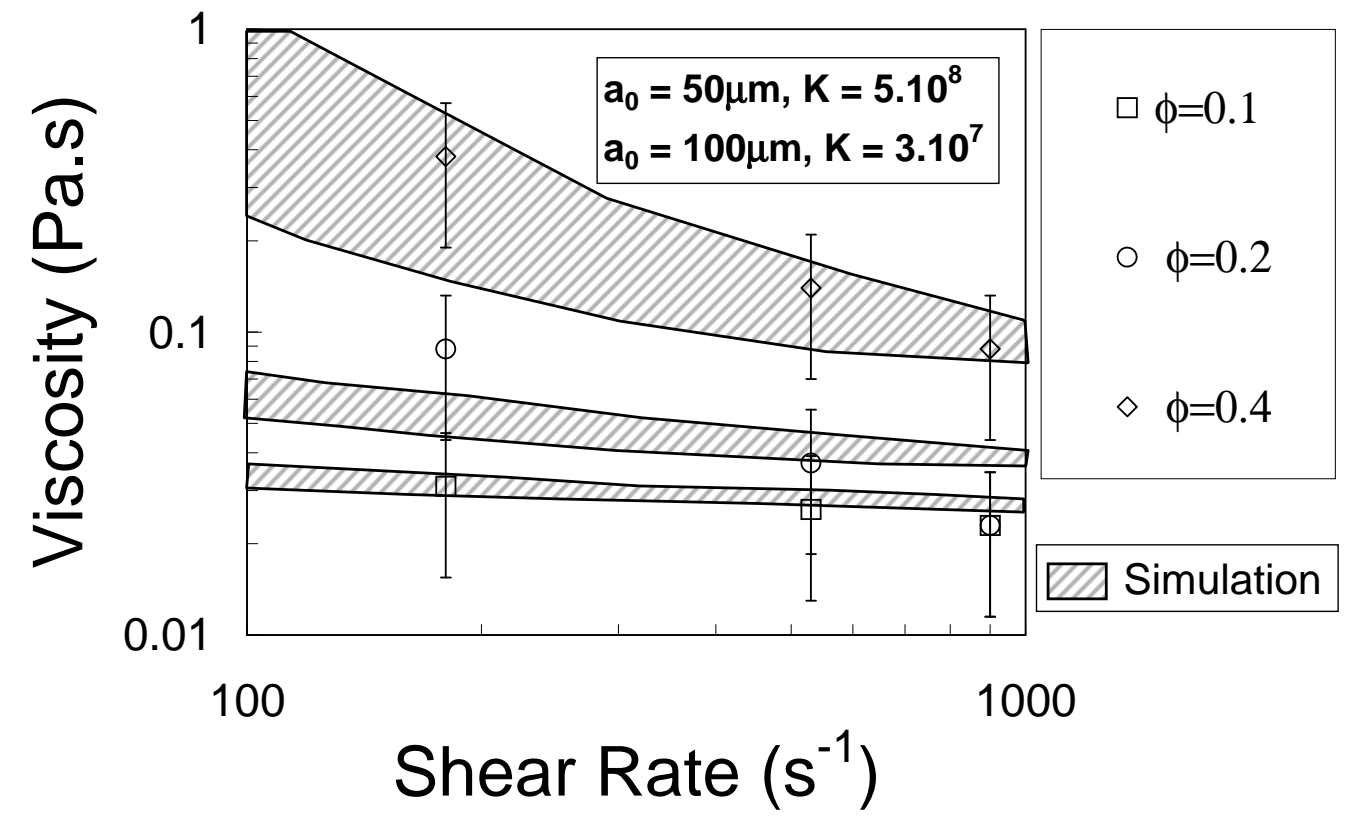

Figure 16: Comparison between simulation results and experimental viscosity measurement of an $A l-6.5 w t \% S i$ alloy [8] as a function of the shear rate and solid fraction. Simulation results are spread over a domain limited by two reasonable values of $a_{0}$. This domain contains the experimental steady state measurements. 


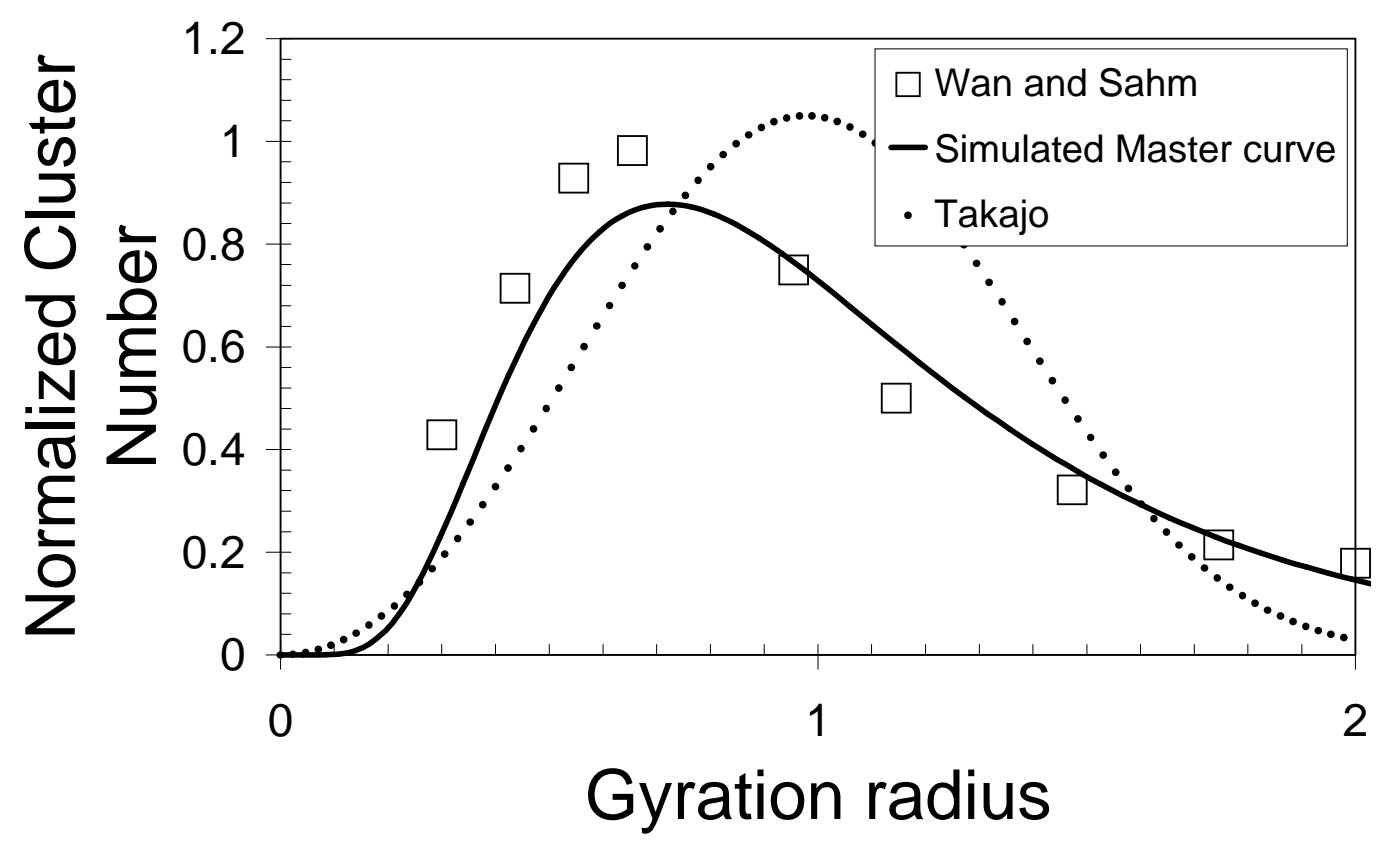

Figure 17: Normalized cluster size distribution: comparison between experimental microstructure analysis of Wan [19], and the present simulation. Result of Takajo [24] are plotted for comparison. The simulated distribution seem to fit accurately the real distribution profile. 